\section{Du bist mir nicht egal}

\section{Kirchgemeinden als Caring Communities ${ }^{1}$}

\section{Dörte Gebhard}

\section{Ich und Ihr}

Jeder Zeitgeist muss kompensieren, muss seine Einseitigkeiten ausgleichen. In einer Epoche der Eigenwilligkeiten, der Individualisierung und der Privatisierung vieler gesellschaftlicher Herausforderungen kommen gemeinsame Bewältigungsstrategien neu ins Blickfeld. Dazu gehören das Verständnis und die Aufgabe, Caring Communities (CCs), gegenseitig fürsorgende Gemeinschaften zu bilden.

Den Kirchgemeinden und der Diakonie kommt zugute, was manchmal hinderlich erscheint: ihr enormes Beharrungsvermögen im rasanten Wandel der Zeiten. Der jüngst gefundene Name CC ist gewiss noch manchen Kirchgemeinden fremd, das Ansinnen aber seit Jahrtausenden vertraut. Im Matthäusevangelium wird in gebotener Kürze ausgemalt, was eine menschliche, verantwortliche und lebendige Gemeinschaft ausmacht, in der der eine der anderen nicht egal ist und umgekehrt - Mt 25, 35-36.402:

Ich war hungrig, und ibr habt mir ₹u essen gegeben.

Ich war durstig, und ibr habt mir zu trinken gegeben.

Ich war ein Fremder, und ibr habt mich als Gast aufgenommen.

Ich war nackt, und ibr habt mir Kleider gegeben.

1 Überarbeiteter Vortrag an der «Impulstagung der Reformierten Landeskirche Aargau für Mitarbeitende in der Seniorenarbeits am 4. Mai 2018 in Aarau.

2 Übersetzungen: Basisbibel, Stuttgart 2012.

Jahrbuch Diakonie Schweiz 3 (2019) - ISSN 2504-3994

Dieser Text ist lizenziert unter einer Creative Commons Namensnennung 4.0 International Lizenz (CC BY 4.0): (https://creativecommons.org/licenses/by/4.0/).
Ich war krank, und ihr habt euch um mich gekümmert.

Ich war im Gefängnis, und ibr habt mich besucht.

Amen, das sage ich euch: W as ihr für einen meiner Brüder oder eine meiner Schwestern getan habt - und wenn sie noch so unbedeutend sind-, das habt ibr für mich getan.

Ein Vorteil dieses Textes ist, dass er weder von vornherein noch alsbald oder gar letztlich festlegt, wer «ich bin und wer «hrs seid. So ist die potentielle Gegenseitigkeit aller menschlichen Hilfsbeziehungen grundsätzlich gewahrt. Sodann bleiben alle Beteiligten vor einem Ranking hinsichtlich Bedeutung und Wichtigkeit einzelner Individuen bewahrt. Drittens fällt auf, dass einer Person von einer Mehrzahl Menschen geholfen wird, jedoch keineswegs vielen oder gar allen Notleidenden von einem Einzelnen geholfen werden muss. Diese Fehlinterpretation liegt in Zeiten der Individualisierung nahe und hat zu einer Geschichte der unmenschlichen Überforderungen, auch in der Diakonie, geführt. Sie sollte nicht fortgeschrieben werden. ${ }^{3}$ Es gibt gute Gründe, immer noch oder wieder von «sorgenden Gemeinschaften» zu sprechen.

\section{2. «Sorge» da und hier}

Eine populäre Definition fasst zusammen: «Der Begriff Sorge beschreibt ein durch vorausschauende Anteilnahme gekennzeichnetes Verhältnis des menschlichen Subjektes zu seiner Umwelt und zu sich selbst. Eine subjektiv erwartete Not (Bedürfnis, Gefahr) wird gedanklich vorweggenommen

3 Vgl. Dörte Gebhard, Menschenfreundliche Diakonie. Exemplarische Auseinandersetzungen um ein theologisches Menschenverständnis und um Leitbilder, NeukirchenVluyn ${ }^{2} 2000,219-222$ 
und wirkt sich im Fühlen, Denken und Handeln des Besorgten oder Sorgenden aus. Das Spektrum reicht dabei von innerlichem Besorgt- oder Beängstigt-Sein bis zur tätigen Sorge für oder um etwas.» ${ }^{4}$

Aber kein althergebrachtes Wort wird heute gesprochen oder geschrieben, das nicht mit schweren Sorgen, Vorurteilen und scheinbaren Entwöhnungsbedürftigkeiten aus der Wirkungsgeschichte belastet wäre. Der «Sorge» geht es nicht anders als z. B. Gott und der Liebe.

In zwei Hinsichten ist die Sorge mit Sorgen beladen. Es geht um ihr Verständnis im Vergleich zum Geld und bei Goethe.

Aus einer verengt-ökonomistischen Sicht wirkt der Begriff nicht operationalisierbar, kaum prüfbar und demzufolge überwindungspflichtig.

«Konnotiert ist der Sorgebegriff [...] mit einem patriarchalen und autoritären Fürsorgeverständnis, das lange Zeit prägend war.» ${ }^{5}$ Der Praktische Theologe Thomas Klie hält dennoch und erst recht am Begriff Sorge fest, mag er auch noch so «betulich» und «altmodisch» erscheinen. Denn «die Sorge um den Anderen und auch das Glück des Anderen sind zentrale Dimensionen unserer Existenz. Wir dürfen 〈Sorge` für Menschen, die der Unterstützung bedürfen, nicht zu einer Dienstleistung reduzieren, die hierzu erforderliche Organisation nicht nur als Sozialtechnik begreifen. Das Thema hat sehr viel mit den Grundfragen des Lebens und unseres sozialen Miteinanders zu tun. Hannah Arendt verweist darauf, dass die soziale und gesellschaftliche Bezogenheit des Menschen zum Kern menschlicher Existenz gehört.. ${ }^{6}$

Entsprechend harsch fällt Klies Urteil über diejenigen aus, die der Ökonomisierung aller Lebensbereiche auf den Leim gehen und Menschen

$-$

4 Art. Sorge, URL: https://de.wikipedia.org/wiki/Sorge, (abgerufen am 25.02.19).

5 Thomas Klie, Caring Community - leitbildfähiger Begriff für eine generationenübergreifende Sorgekultur?, in: Sorgende Gemeinschaften - Vom Leitbild zu Handlungsansätzen. Dokumentation. ISS im Dialog. Fachgespräch am 16. Dezember 2013 in Frankfurt am Main, 13

6 Ebd. nur noch beschränkt wahrnehmen, etwa, wenn nur noch von Kunden die Rede ist: «Anteilnehmende, vorausschauende Verantwortungsübernahme heißt wesentlich mehr als DIN ISO-Qualität. Nichts gegen gute Dienstleistungen. Sie können aber nur einen bescheidenen Beitrag zu dem liefern, was die Sorge füreinander ausmacht. Es ist unverständlich für mich, dass sich etwa ein Diakonisches Werk oder eine Arbeiterwohlfahrt so auf das ökonomische Paradigma des Kunden verlegt haben und Menschen, denen ihre Solidarität gilt, auf Dienstleistungsempfänger reduziert haben. Was ist in dieser Gesellschaft nur schiefgelaufen, dass man auch noch stolz darauf ist, gute Noten für die Pflege zu bekommen? Verbraucherschutz ist alles? Das ist anthropologisch primitiv, sozialpolitisch verfehlt und aus Sicht einer strategischen Zukunftssicherung der freien Wohlfahrtspflege gefährlich. Diese Fehlentwicklung gilt es dringend zu korrigieren, ohne dabei sozialstaatliche Verpflichtungen und Garantiefunktionen zu relativieren. Die Wiederentdeckung der «Sorge» im zivilgesellschaftlichen und politischen Diskurs kann wichtige Reflektionsprozesse anstoßen. Hierin liegt die Irritationsqualität des Begriffs, die ihn mit sympathisch macht.» ${ }^{7}$

Schon das Nachdenken über CCs stiftet Hoffnung, dass die trendkritischen Kräfte in Kirche und Diakonie mehr Aufmerksamkeit und Gehör finden. Isolde Karle plädiert angesichts einer «Kirche im Reformstress» für Weitblick - bis hin zu einem «Gegenhorizont».

«Durch die Ökonomisierung der Kirche entsteht eine Eigendynamik der Organisation, die sich theologischen Beurteilungskriterien mehr und mehr entzieht. An die Stelle theologischer Steuerung tritt immer stärker eine managementförmige Steuerung. Theologie wird zur legitimierenden Zweitcodierung. Für die Kirche der Zukunft ist es unabdingbar, dass sie wieder zu einem eigenen theologischen Selbstverständnis findet, dass sie religiös sprachfähig ist und sich als Organisation nicht von den Zwängen

7 Ebd.
Jahrbuch Diakonie Schweiz 3 (2019) - ISSN 2504-3994

CC by 4.0
Jahrbuch Diakonie Schweiz 3 (2019) http://dx.doi.org/10.22018/JDS.2019.3 
ökonomischer Logik fremdbestimmen lässt. Die Kirche ist Teil der Gesellschaft und zugleich Gegenhorizont zu einer durchrationalisierten, leistungsorientierten Welt. Sie symbolisiert das Unverfügbare, nicht Messund Berechenbare und darin das Angewiesensein auf Gottes Güte, Gnade und Erbarmen.. ${ }^{8}$

Der Begriff «Sorge» hat aber noch eine andere, irrtierende Dimension, die seit Johann Wolfgang von Goethe leider prägend ist. Sorge erschien seinerzeit bei ihm als ein verfinsterndes, jegliches Verantwortungsbewusstsein raubendes, vergeblich Energie fressendes Wesen, das unter keinen Umständen zu sozialen Handlungen befähigt oder auch nur ermuntert. Der wohlsituierte, reiche Mann Faust bekommt Besuch von der Sorge, die durch jenes Schlüsselloch dringt, wo «Not», «Mangel» und «Schuld» vor verschlossenen Türen stehen. Um die Grösse und Weite der Antipathien gegenüber Sorgen vieler Art ermessen zu können, sei hier der Sorge definierende Abschnitt aus Goethes «Faust» zitiert:

$$
\text { «Sorge }
$$

Würde mich kein Obr vernehmen,

Müßst' es doch im Herzen dröhnen.

In verwandelter Gestalt

üb' ich grimmige Gewalt.

Auf den Pfaden, auf der Welle,

Ewig ängstlicher Geselle,

Stets gefunden, nie gesucht,

So geschmeichelt wie verflucht. -

Hast du die Sorge nie gekannt?

$$
\text { [...] }
$$

Wen ich einmal besitze,

Dem ist alle Welt nichts nütre;

8 Isolde Karle, Kirche im Reformstress, Gütersloh 2010, 256.
Ewiges Diistre steigt herunter,

Sonne gebt nicht auf noch unter,

Bei vollkommnen äßern Sinnen

Wobnen Finsternisse drinnen,

Und er wei $\beta$ von allen Schätzen

Sich nicht in Besitz, zu setzen.

Glück und Unglück wird sur Grille,

Er verbungert in der Fülle;

Sei es Wonne, sei es Plage,

Schieb er's zu dem andern Tage,

Ist der Zukunft nur gewärtig

Und so wird er niemals fertig.»"

Gegen den Verdacht, Sorge könne immer nur ängstliche Antizipation des Ungewissen bedeuten, ist in der Bergpredigt die Anweisung zu finden: Sorgt euch nicht (Mt 6,25) mit ausführlichen und anschaulichen Begründungen (Mt 6,25-34)

Vor allem aber ist hervorzuheben, dass die gegenseitige Sorge und Verantwortung nach Matthäus 25 unmittelbar zur Tat schreitet. Eine Caring Community wäre missverstanden als Versammlung besonders furchtsamer Zeitgenossen im Gefolge Goethes, die sich der vergeblichen $\mathrm{Zu}-$ kunftsvermeidung widmen.

Der Auftrag für CCs ist die aktive Gestaltung und Veränderung der Gegenwart.

\section{Wer sind wir?}

Gemeinschaften, in denen in Wort und Tat gilt: «Du bist mir nicht egal», fallen nicht vom Himmel, sondern wachsen und gedeihen nur über lange

9 Johann Wolfgang von Goethe, Faust II, 5. Akt. 
Zeiträume. Solche Gemeinschaften müssen zunächst wahrgenommen, dann beschrieben, erklärt und begründet werden, müssen drittens überliefert und viertens ständig reformiert werden. Zur Illustration unterbreche man am besten die Lektüre des Jahrbuchs Diakonie beim nächsten Absatz und teile ein weisses Blatt Papier in zwei Hälften. Dann notiere man auf der einen Seite alle Menschen, die einem nicht egal sind und auf der anderen Seite all' jene, denen man seinerseits nicht egal ist.

Dieser zunächst höchst individuelle Befund fördert hoffentlich ein tragfähiges Netz von Beziehungen und Kommunikations- und Hilfsmöglichkeiten auf beiden Seiten zutage. Aber auch extreme Einseitigkeiten werden so der Selbsterkenntnis zugänglich. Das Ergebnis kann ohne Umstände weiteren Fragen unterzogen werden: Sorge ich als Frau oder Mann für mehr Männer mehr oder Frauen? Bin ich verantwortlich für mehrere Altersgruppen oder (nur) für eine? Bin ich spezialisiert auf Kranke oder Gefangene, auf Einheimische oder Flüchtlinge, auf Hungrige an Leib und/oder Seele? Warum? Welche Ähnlichkeiten haben die Menschen, die nach mir schauen? Kennen sie sich untereinander? Kommunizieren oder organisieren sie gar ihr Tun? Und wie tragen sie sich und ich mir selbst Sorge, wie ein genialer Helvetismus der deutschen Sprache präzis formuliert?

Impulsgebend ist diese Übung in Kleingruppen bzw. in überschaubaren Gemeinden und Gemeinschaften, die schon Caring Communities sind oder (wieder) werden wollen.

So oft diese Übung bisher vollzogen wurde, haben sich wahrhaft erbauliche und tragfähige Zusammenhänge gezeigt, wenn ein weiteres betuliches und wie aus der Zeit gefallenes Wort eingeführt werden darf.

Erbaulich ist der Blick auf das bereits Vorhandene, gerade wenn und insofern es überschaubar ist.

Der Überschaubarkeit von entstehenden oder bereits etablierten CCs ist besondere Aufmerksamkeit zu widmen, da bei aktuellen kirchlichen Reformen regelmässig der entgegengesetzte Trend zu beobachten ist. Um ökonomischen Leitbildern zu genügen, werden Räume und Strukturen in Grössenordnungen geschaffen, die für die Einzelne nicht zu übersehen sind und sich der verantwortlichen Gestaltung, selbst durch hoch motivierte Mitarbeitende und Freiwillige, entziehen.

Isolde Karle hebt in ihren «Zwölf Thesen zur Kirchenreform» hervor: «Die evangelische Kirche wächst aus den Gemeinden, den lokalen Zusammenschlüssen von Christinnen und Christen. Kleinere Einheiten kommen im Gegensatz zu größeren Einheiten mit wenig bürokratischer Kontrolle aus, weil die persönliche Bekanntschaft und der überschaubare Rahmen eine starke Vertrauensbasis schaffen. Die Vertrautheit von Orten und Personen hat eine kaum zu überschätzende Funktion für die Vermittlung elementarer Grundsicherheit. Die Bedeutung solcher Grundsicherheit nimmt mit der Anonymität und Mobilität der Gesellschaft eher zu statt $\mathrm{ab.} .{ }^{10}$

Jede menschenfreundliche Gemeinschaft muss absehbare Grenzen haben: lokal, regional und weltweit. Nur eine bestimmte Zahl an Kolleginnen und Kollegen ist zu koordinieren, nur eine endliche Zahl von Gemeindegliedern ist in Leben und Glauben sinnvoll zu begleiten, nur ein begrenztes Mass an Netzwerkarbeit kann geleistet werden, damit Gemeinschaften hilfreich, stabil und offen für Neue(s) agieren können.

\section{Nicht egal - dir und mir}

Gemeinschaften, in denen in Wort und Tat gilt: «Du bist mir nicht egal», zeichnen sich durch Zusammengehörigkeit und einen gemeinsamen Horizont, aber gerade nicht durch möglichst hohe Homogenität aus. Die aktuelle Herausforderung, dass einer des anderen Last trage (vgl. schon Paulus in Gal 6,2), ist durch Wahrnehmung und Gestaltung von Vielfalt leichter zu bewältigen. Gerade weil wir nicht alle dieselben Lasten zu tragen haben, können wir diakonisch tätig werden, einander beistehen, helfen: Alte und Junge, mit und ohne Behinderung, mit und ohne Demenz,

10 Karle, Kirche (Anm. 8), 256.
Jahrbuch Diakonie Schweiz 3 (2019) - ISSN 2504-3994

CC by 4.0
Jahrbuch Diakonie Schweiz 3 (2019) http://dx.doi.org/10.22018/JDS.2019.3 
mit und ohne Arbeit, mit und ohne Wohnung, mit und ohne Aufenthaltsbewilligung, mit und ohne Zeit. Diese Liste ist selbstverständlich nich vollständig. Folgende These wird mit ihr exemplarisch erläutert: Um Pluralität, Diversität nicht nur zu ertragen, sondern zu nutzen und zu geniessen, braucht es um einer tatkräftigen, gemeinschaftsstiftenden Sorge willen Mut.

Ein langjähriger Bewohner in einer diakonischen Einrichtung in Deutschland, ein Mensch mit geistiger Behinderung, wie wir gewöhnt sind zu sagen, verfasste auf die Frage, was Diakonie sei, u.a. die folgenden Wortreihen in grossen Buchstaben und rheinischer Mundart:

$\begin{array}{ll}\text { MUT } & \text { SCHAFTSHILFE } \\ \text { MUT } & \text { FROINDLISCH } \\ \text { MUT } & \text { FROINDLISCHKEIT } \\ \text { MUT } & \text { FRUNDEN HABEN } \\ \text { MUT } & \text { INHERLISCHT } \\ \text { MUT } & \text { FROIDSCHAFTSPREIS } \\ \text { MUT } & \text { IHRWEGHEN } \\ \text { MUT } & \text { LEISTUNK }\end{array}$

Was jener Mensch über die Diakonie seinerzeit an Gedanken zusammentrug ${ }^{11}$, ist anwendbar auf Kirchgemeinden als diakonisch zusammenwirkende Caring Communities.

11 Vgl. zur Auslegung der gesamten Wortreihe Dörte Gebhard, ErMUTigung - Zur theologischen Begründung diakonischen Handelns, in: Reinhard Schmidt-Rost/Norbert Dennerlein/Udo Hahn (Hg.), Soll ich meines Bruders Hüter sein? Erkundungen und Reflexionen zum spannungsreichen Verhältnis von Kirche und Diakonie, Hannover 2005, 51-63.
Was geschieht in einer CC? Gegenseitige Hilfe in einer überschaubaren Gemeinschaft, die Mut schafft, kurz Mutschaftshilfe. Ob es eine Zeittauschbörse im Quartier, eine regional organisierte niederschwellige Alltagshilfe wie «KISS - Keep it small and simple. Begleitete Nachbarschaftshilfe» ${ }^{12}$, ein Besuchsdienstkreis oder eine kirchgemeindliche Seniorenwandergruppe mit Einsamkeitsprophylaxe als erwünschter Nebenwirkung ist - jede Hilfe muss sich daran messen lassen, ob sie Mut und Freiheit, Flexibilität, Freiraum und Freizeit schafft oder raubt. Der Philosoph Odo Marquard hat CCs - ohne diesen Begriff zu kennen - im Blick auf geteiltvermehrte Zeiten bereits von vornherein gelobt:

«Gerade weil wir trotz der Einzigkeit unserer Lebenszeit mehrere viele - Lebenszeiten brauchen, brauchen wir unsere Mitmenschen: Die Kommunikation mit ihnen ist für uns - zeitmangelkompensatorisch - die Chance, trotz der Einmaligkeit unseres Lebens viele Male zu leben, also trotz der Einzigkeit unserer Lebenszeit viele Lebenszeiten zu haben, die zugleich z. B. weil diese Lebenszeiten der Anderen teils vor unserer Lebenszeit beginnen und teils nach unserer Lebenszeit aufhören oder mit Lebenszeiten verflochten sind, die vor unserer eigenen Lebenszeit beginnen und nach ihr aufhören - unsere kurze Zeit länger machen. Das Mitsein mit den Mitmenschen ist - wenn der Mitmensch nicht gerade ein Zeitdieb ist, der Zeit nur stiehlt (was man übrigens, fürchte ich, auch durch öffentliche Vorträge tun kann) - für uns die Möglichkeit, mehr Zeit zu haben als wir haben: denn geteilte Zeit ist vielfache Zeit. Diese Pluralisierung unserer Lebenszeit - als Lebenspluralisierung - brauchen wir; und wir bekommen sie von unseren Mitmenschen, und zwar durch das, was man nennen kann: die mitmenschliche Multitemporalität. Mit so vielen

12 Vgl. z. B. KISS - Nachbarschaftshilfe mit Zeitgutschriften; URL: https://kiss-reusstalmutschellen.ch (abgerufen am 12.03.19).
Jahrbuch Diakonie Schweiz 3 (2019) - ISSN 2504-3994

CC by 4.0
Jahrbuch Diakonie Schweiz 3 (2019) http://dx.doi.org/10.22018/JDS.2019.3 
Mitmenschen einer als Mitzeitler kotemporiert, so viel mal ist er ein Mensch: durch diese Multitemporalität, die die Menschlichkeit steigert.» ${ }^{13}$

Grosse Investitionen von Zeit rechnen sich jenseits der finanziellen Dimension. Die Gründerinnen und Gründer von KISS erläutern die absichtlich geldfreie Mutschaftshilfe so: «Freiwillige helfen einander in der Nachbarschaft. Dies stärkt den Zusammenhalt und macht Freude. Die Freiwilligen können ihre eingesetzten Stunden gutschreiben. Alle Genossenschafter sind Gebende und Nehmende und begegnen sich auf Augenhöhe. Dies führt zu hoher Zufriedenheit und Anerkennung bei den Mitgliedern. Ziel ist, dass Menschen aller Generationen die Betreuung benötigen, durch Nachbarschaftshilfe möglichst lange zu Hause in ihrem gewohnten Umfeld bleiben können. KISS arbeitet schweizweit am Aufbau der 4. geldfreien Vorsorgesäule. KISS bedeutet «Keep it small and simple〉. Eine überschaubare Gruppe von sich vertrauenden Menschen (small) geben und nehmen auf einfache unbürokratische Art (simple) Unterstüt zung. [...] In der Genossenschaft vor Ort wird Gemeinschaft gelebt. Dort treffen sich die Engagierten und Interessierten, tauschen Erfahrungen und Wissen aus, unterstützen sich gegenseitig, entwickeln Ideen und Zusammengehörigkeit.» ${ }^{14}$

Wo Mutschaftshilfe in und über Kirchgemeinden hinaus geleistet wird, ist auch der Graben zwischen Seelsorge und Diakonie überwindbar. Ausserdem drückt sich in diesem Begriff die grundlegende Ressourcenorientierung gegen eine allzu verbreitete Defizitsuchsucht aus.

Mutschaftshilfe begegnet in der Bibel, natürlich ohne diesen Begriff, in zahlreichen Zusammenhängen. Jesus heilt Menschen und integriert sie in alte oder neue Gemeinschaften.

Dabei kommt es auf «Froindlischkeit» an, wie gleich zwei Zeilen lang hervorgehoben wird.

13 Odo Marquard, Zeit und Endlichkeit, in: Ders., Skepsis und Zustimmung, Stuttgart $1994,45-58,56 f$

14 URL: https://kiss-reusstal-mutschellen.ch (abgerufen am 12.03.19).
Freundlichkeit als zugrundeliegende Haltung ist natürlich ein Containerbegriff, der aber geeignet ist, vieles zu fassen: biblische Sanftmu (Mt 5,5) und neuzeitliche Toleranz, trendige Empathie und Shalom, umfassender Frieden, in bereits jüdischer Perspektive.

Jede CC hat Mut zur Toleranz nötig. Damit ist gerade nicht eine fortschreitende Vergleichgültigung der Motive für soziales Tun gemeint. Mutige Toleranz impliziert die aktive Auseinandersetzung mit dem zu Tolerierenden, nicht eine gefährliche Mischung aus Unwissenheit und Desinteresse. Toleranz meint aktives Ertragen und - speziell für Christen - aktives Erleiden von Widersprüchen, weil sich die gute Nachricht von der Liebe Gottes gerade nicht mit Gewalt durchsetzen lässt. Konfrontationen und Konflikte, herkommend aus kultureller Diversität, gehören zu CCs, wenn sie sich nicht von vornherein selbst ghettoisieren. Die «Konfrontation mit mir fremden Glaubensweisen [ist] nicht als göttlicher Aufruf zur Relativierung der eigenen Glaubensbindung zu verstehen, sondern als Ansporn, sich des eigenen Glaubens zu vergewissern und diesen umso bewusster zu leben.» ${ }^{15}$ Mutige Toleranz setzt also nicht nur Pluralität, sondern zugleich und erst recht Positionalität voraus. Verständigung erfordert Identitäten, Kommunikation ist das Lebenselixier für CCs. Daher ist es notwendig, «Frunden» zu haben, eine schöpferische Wortkombination aus «Freude» und «Freunde».

Kirchgemeinden sind das Gegenteil von homogenen Gruppen; Menschen aus den unterschiedlichsten religiösen, sozialen und politischen Milieus begegnen sich, nicht nur aus bis zu fünf verschiedenen Generationen. Jeder Mensch, der sich in einer CC engagiert, muss mit gutem Willen und Wohlwollen, intrinsischer Motivation und aktivem Engagement jenseits der Grenzen der eigenen Glaubens- und Gewohnheitswelt rechnen. Im

15 Ulrich H. J. Körtner, Vielfalt und Verbindlichkeit. Christliche Überlieferung in der pluralistischen Gesellschaft: Forum Theologische Literaturzeitung 7, Leipzig 2002, 105 
biblischen Bild aus dem Lukasevangelium (vgl. Lk 10,25-37): Der von einem Fremden geschlagene Israelit muss der Hilfe des ebenso fremden Samariters vertrauen, sobald er wieder bei Bewusstsein ist. Auch innerhalb von CCs, die sich in, mit und durch Kirchgemeinden bilden, ist die Privatisierung, Individualisierung und Pluralität von ethischen und religiösen Vorstellungen gross - die passenden Stichworte in der Liste heissen «inherlischt», also «innerlich» und «ihrweghen», also «um ihretwillen».

Um wessentwillen?

Um Himmels willen?

Um Gottes willen? Es gibt wohl niemanden mehr, der sich in CCs engagiert, um sich das Himmelreich zu verdienen. Man hilft und hofft, dass es den Nächsten und auch einem selbst zugute komme.

Mutschaftshilfe wird nur funktionieren, wenn man «Frunden», also Freude und Freunde hat, d.h. wenn Mitarbeitende und Hilfesuchende im tieferen Sinne des Wortes zusammenarbeiten können, weil ihnen die Grundintentionen behagen, klar und vertraut sind. Da sich aber nichts von vornherein von selbst versteht, z. B. dass die Pflege der Gottebenbildlichkeit etwas anderes ist als die Wiederherstellung der Arbeitsfähigkeit, dass die Rechtfertigung aus Gnade nicht geraden Weges in Fatalismen führt, weil man glaubt, dass man nichts machen kann und daher auch nichts mehr machen braucht ..., dass Krankheiten keine Strafen Gottes sind (vgl. Joh 9,1-3) und dass die Unterscheidung zwischen Person und Werk den Unterschied zwischen Seelsorge bzw. diakonischem Beistand und juristischen Maßnahmen deutlich macht ... - aus all diesen und unzähligen Gründen mehr, können CCs nicht ohne Kommunikation, nicht ohne permanenten Austausch auskommen. Die Evidenz von sich entwickelnden Werten und Zielen kann nicht stillschweigend vorausgesetzt werden, sondern nur in ehrlichen Auseinandersetzungen zu Konvergenzen geführt werden. Daher sind CCs mehr als eine organisatorische Herausforderung. Sie sind die seltene Gelegenheit in unserer Gesellschaft, den je eigenen Glauben in seinen Konsequenzen zu kommunizieren:
Seid allezeit bereit zur Verantwortung vor jedermann, der von euch Rechenschaft fordert über die Hoffnung, die in euch ist, und das mit Sanftmut und Gottesfurcht (1 Petr 3,15).

Die Entstehung jeder CC hat einen «Froidschaftspreis», einen hohen, jedoch nicht finanziellen Preis. Thomas Klie hat die Herausforderungen für politische Gemeinden zusammengetragen, sie lassen sich leicht für Kirchgemeinden anverwandeln:

\section{«Dazu gehören:}

die Überwindung einer Logik der Ökonomisierung aller Lebensbereiche,

die Überwindung eines anachronistisch-romantischen Familialismus,

die Praxis einer neuen Gastfreundschaft gegenüber dem Homo Patiens (als Bereitschaft zur Gabe und zum Teilen mit Fremden sowie in der Überwindung von Angst, Distanz, Ekel und anderen Formen der Abgrenzung),

die Offenheit für genossenschaftliche Antworten auf Voraussetzungen für Bedingungen der Existenz vor Ort (Daseinsvorsorge) sowie

die Entfaltung einer Innovationskultur (Leitbilder, Strukturen, Befähigung).» ${ }^{16}$

Auch in Kirchgemeinden muss die einseitige Aufmerksamkeit für ökonomische Sachverhalte regelmässiger hinterfragt werden, spielen gegensätzliche Auffassungen von Familie eine erhebliche Rolle, ist die christliche Gastfreundschaft immer wieder zu üben, kann die Offenheit für genossenschaftliche Projekte nicht in allen Milieus gleichermassen gegeben sein und herrscht örtlich schwere Erschöpfung, weil schon zu viele Leitbild- und Strukturmassnahmen ohne innovative und v.a. ohne theologische Kultur dahinter durchgeführt worden sind. Aber auch ein effektiver und harmonischer Leitbild- bzw. Genossenschaftsgründungsprozess darf nicht darüber hinwegtäuschen, dass Leid und Hoffnungslosigkeit in der

16 Klie, Caring community (Anm. 5), 20.
Jahrbuch Diakonie Schweiz 3 (2019) - ISSN 2504-3994

CC by 4.0
Jahrbuch Diakonie Schweiz 3 (2019) http://dx.doi.org/10.22018/JDS.2019.3 
Welt eine ernsthafte Anfechtung des christlichen Glaubens und damit auch jeglicher Arbeitsmotivation sind und bleiben.

So bleibt Mutschaftshilfe in CCs unter allen irdischen Umständen auch ohne ökonomistische Einseitigkeiten eine «Leistunk». Der Samariter hat dem Wirt eine Fallkostenpauschale gezahlt und sich bereiterklärt, auf Antrag freiwillig eine Zusatzleistung zu erbringen (Lk 10,35). Bis zu diesem Moment, in dem er den Samen für eine kleine CC aussät, hat er schon ein beträchtliches Mass an Lebenszeit aufgewendet und nicht verrechnet. So wird es auch in der Gegenwart sein. Zeit wird immer mehr wert sein als Geld, besonders in Zeiten, in denen angeblich Zeit Geld ist. ${ }^{17}$ Aber jeder Zeitgeist kann kompensieren, kann seine Einseitigkeiten ausgleichen. Dazu gehören für zeitgenössische Kirchgemeinden das Verständnis und die Aufgabe, eine CC, eine gegenseitig fürsorgende Gemeinschaft zu werden.

Autorin:

Dörte Gebhard, PD Dr. theol., Pfarrerin und Privatdozentin für Praktische Theologie an der Universität Zürich

17 «Zeit ist Geld» begegnet bei Benjamin Franklin bereits 1748! 